\title{
Mental illness stigma: Strategies to address a barrier to care
}

\section{By Teresa M. Conklin, DNP, APN, FNP-BC}

Mental illness (MI) is extremely prevalent worldwide. Despite recent efforts to raise awareness through screening and early identification, $\mathrm{MI}$ stigma remains high and is recognized as a major barrier to recovery. Nurse practitioners play a key role in overcoming stigma to facilitate appropriate treatment and recovery. The various aspects of MI stigma, tools to measure stigma, and intervention strategies to decrease stigma among healthcare professionals and individuals suffering from $\mathrm{MI}$ are discussed. Disparities between women and men are also reviewed.

KEY WORDS: mental illness, mental illness stigma, healthcare stigma, self-stigma, social stigma

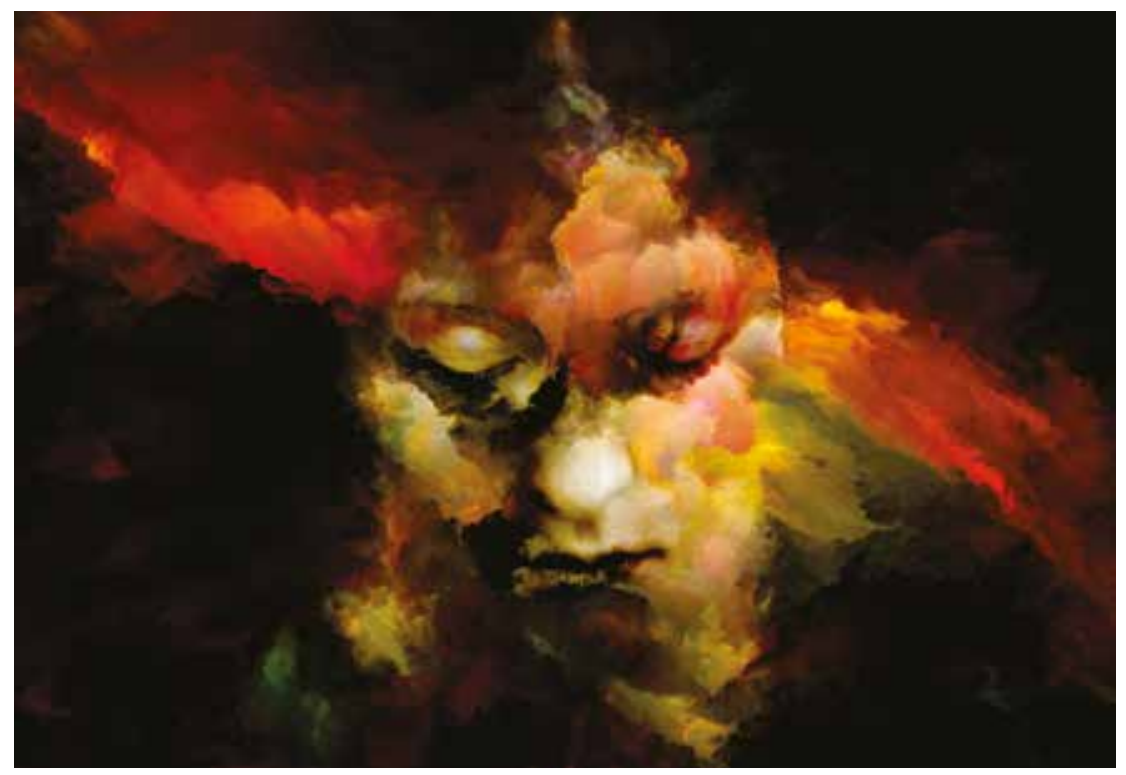

$\mathrm{M}$ ental illness (MI) affects 1 in 5 adults in the United States and is a leading cause of morbidity and disability. ${ }^{1}$ Although it does not discriminate based on race, ethnicity, socioeconomic status, or gender, the prevalence of $\mathrm{Ml}$ is greater in women than men $(22.3 \%$ vs $15.1 \%){ }^{2}$ Women are more likely to suffer from serious mental health problems resulting in profound functional impairment and disability when compared with men $(5.7 \% \text { vs } 3.3 \%)^{2}$ In addition, more women attempt suicide than men. ${ }^{3,4}$ Despite the high prevalence and severity of $\mathrm{Ml}$ among women, most will never receive professional mental health services, which can negatively impact every aspect of their lives. ${ }^{2}$

Many factors contribute to inadequate utilization of mental health services including personal and public beliefs about MI. Negative perceptions influenced by lack of knowledge, past experiences, media portrayal, and societal norms can lead to discrimination and stigma. Although stigma is just one of many factors that can impede access to mental health services, it is one that has profound effects on outcomes and recovery for those suffering. By recognizing the impact of Ml stigma 
Figure. America's mental health 2018 survey results (barriers to mental health services as identified

by respondents) ${ }^{7}$

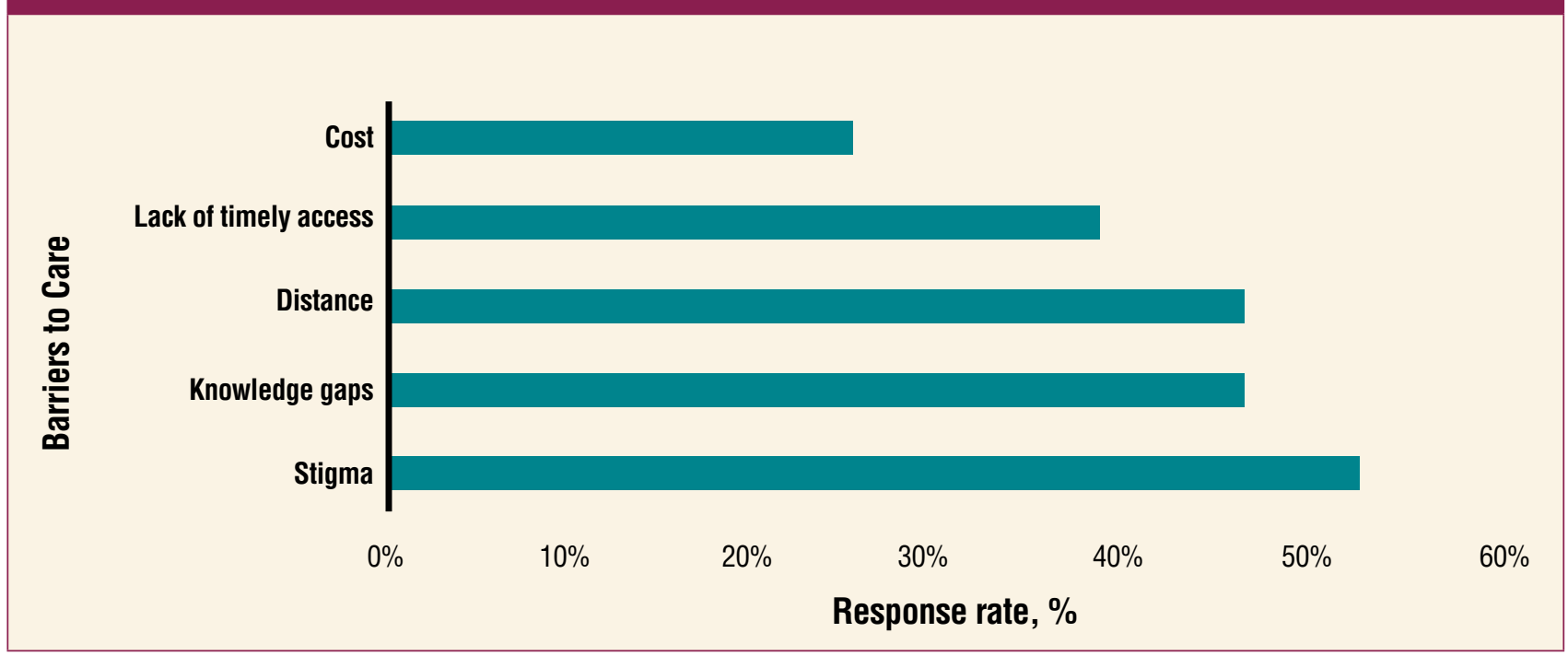

and implementing stigma reduction measures, more individuals suffering with Ml will get the care they need, making recovery much more likely.

Although screening and identification of MI has increased in recent years, current practices fail to meet the treatment needs of those affected. ${ }^{5,6}$ The World Health Organization and other professional groups have identified barriers to care. These include stigma, lack of knowledge, discrimination, lack of understanding, lack of awareness, cost, distance, lack of timely access, and insufficient intervention strategies that integrate primary healthcare and mental health services. ${ }^{1,3,7}$ Attitudes about $\mathrm{Ml}$ and access to mental health services were assessed in a comprehensive study conducted by the National Council for Behavioral Health and Cohen Veterans Network. The two-pronged research study collected data by surveying 5,000 US adults and analyzing state mental health resources regarding patients' access to mental health services. The results revealed five key barriers to care: stigma, knowledge gaps, distance, lack of timely access, and cost.
The survey found that $52 \%$ of respondents tried to "grin and bear it" when feeling depressed or mentally unstable, noting fear of stigma as a deterrent to seeking care; $46 \%$ never sought care because they did not know where to go; $46 \%$ have had to, or know someone who has had to, travel up to an hour roundtrip to access care; $38 \%$ reported having to wait longer than a week for available services; and $25 \%$ have had to choose between mental health services and paying for routine necessities (Figure). ${ }^{7}$ Without access to effective treatment, individuals with $\mathrm{Ml}$ are predisposed to more physical health problems and a life expectancy that may be reduced by 15 to 20 years when compared to those who receive appropriate treatment. ${ }^{8}$

\section{Mental illness stigma}

Stigma, which is defined as a mark of shame or disgrace that is associated with a certain person, quality, or circumstance, is one of the most fundamental and unceasing challenges that individuals with $\mathrm{Ml}$ face along their journey to recovery. ${ }^{9}$ Three major factors contribute to stigma: lack of knowledge, negative attitudes, and discrimination. ${ }^{10,11}$ Stigma can be associated with a variety of health conditions (eg, HIV, tuberculosis, cancer, obesity), but $\mathrm{Ml}$ stigma remains especially severe and pervasive. ${ }^{10,11}$

Mental illness stigma is multifaceted. Self-stigma and social stigma are the two main contributors. ${ }^{10,12}$ Self-stigma occurs when a person with MI internalizes negative labels, which leads to low self-worth, shame, and hopelessness. The prevalence of self-stigma is high, ranging from $22.5 \%$ to $97.4 \%$ and is thought of as a "second illness" by those suffering. ${ }^{12}$ This internalized negativity permeates all races, age groups, socioeconomic classes, religions, and every type of MI. It is associated with delayed treatment, worsening symptoms, poor recovery, and higher rates of suicide in some cases. ${ }^{12}$ Social stigma refers to the stereotypes that the public assigns to people with $\mathrm{Ml}$ and is associated with discrimination. ${ }^{10}$ The high prevalence of social stigma has been well researched and is linked with impeded access to necessary psy- 
chological support and care. ${ }^{10}$ An international survey found that only $7 \%$ of randomly chosen respondents representing the general population of over 200 countries believed that recovery from $\mathrm{Ml}$ is possible and $98 \%$ of those respondents agreed that individuals with $\mathrm{Ml}$ are stigmatized and discriminated against. ${ }^{13}$ Social stigma can exist within families, religious communities, work environments, social groups, and professional organizations as well as in the place where mental health services are sought, the healthcare setting. ${ }^{10}$ Both social stigma and self-stigma can serve as critical barriers to treatment and recovery.

\section{Stigma in healthcare}

Mental illness-related stigma in healthcare negatively impacts the behaviors of health providers, their work environment, and their ability to facilitate treatment for affected individuals. ${ }^{14}$ Numerous studies suggest that healthcare professionals hold some form of stigma against individuals with Ml. Research demonstrates significant prevalence of MI stigma across the healthcare spectrum and highlights the effects of negative attitudes on those suffering from $\mathrm{Ml}$, often resulting in feelings of rejection and being undeserving of care. ${ }^{14}$ Mental illness stigma among providers is noted to be a roadblock to necessary management and support for those with MI. ${ }^{14}$ Stigmatization in healthcare can occur on multiple planes throughout the system simultaneously. On a structural level (eg, agency policies and practices), there may be a lack of staff training regarding appropriate recognition and/or management of a person who presents with symptoms of MI or impending crisis. On an interpersonal level (eg, patient-provider interactions), there may be discrim- inatory behavior, labeling, negative attitudes, or minimization of symptoms on the part of the provider. ${ }^{14}$ On an intrapersonal level (eg, selfstigma), a patient may be reluctant to seek care or disclose information about their existing or developing MI due to fear or embarrassment. ${ }^{14}$ Any of these factors can prevent access to high-quality care.

Health provider-related MI stigma is often unintentional and subtle. An example would be a provider who, with inadequate knowledge about serious $\mathrm{Ml}$, dismisses a patient with schizophrenia from care due to disruptive behaviors or nonadherence to treatment without recognizing that these are symptoms of a poorly controlled MI. Another example of inadvertent stigmatization might involve a healthcare provider who lacks awareness and delays the medical workup for a middle-aged woman with depression who is "always tired," assuming that her complaints are solely related to depression. Unintentional stigmatization might also occur when a provider minimizes a teenage girl's report of feeling more nervous and anxious, which ultimately results in deferring appropriate referral to a behavioral health practitioner. In a busy office setting, it is easy to dismiss symptoms of Ml when they do not fit into the construct of a typical medical encounter or if they make the provider uncomfortable due to their lack of knowledge or negative attitudes.

Mental illness stigma in healthcare tends to be overlooked and under addressed. Given its damaging effects, it is critical to raise awareness among providers and patients by encouraging self-reflection on attitudes, behaviors, and perceptions. Currently, guidelines have not been established that screen for the presence of stigma. Mental illness stigma can be objectively assessed, however, by using a standardized tool.

\section{Tools}

Many psychometrically sound assessment tools can be used to evaluate stigma. The following are examples of brief, easy-to-use instruments that can be administered to healthcare providers, individuals with $\mathrm{Ml}$, and adults in the general population.

\section{Attitudes to Mental IIIness Questionnaire}

The Attitudes to Mental Illness Questionnaire (AMIQ) is a 5 -item tool that can be used to assess health professionals' attitudes about individuals with various types of MI. ${ }^{15} \mathrm{AMIQ}$ uses a vignette format with a 5-point Likert scale to identify perceived devaluation and discrimination related to those with MI. ${ }^{14}$ It has excellent validity and reliability and can easily be self-administered. ${ }^{15}$

\section{Inventory of Stigmatizing Experiences}

The Inventory of Stigmatizing Experiences (ISE) is a 10-item questionnaire that is used to assess internalized negative attitudes (self-stigma). ISE uses a "never to always" Likerttype scale to measure the prevalence and frequency of stigma as experienced by a person with MI. ${ }^{16}$ It elicits information about the degree to which internalized negativity pervades the various life domains of the affected individual. ${ }^{16}$ This tool has yielded reliable and valid data and can be administered to current or potential consumers of mental health services. ${ }^{16}$

\section{Social Distance Scale}

The Social Distance Scale (SDS) is made up of a 7-item "willingness" scale. The higher the score, the greater the desire of the respondent to distance themselves from an individual within an assigned population (ie, MI). The original version 
dates back to 1925 and has undergone considerable modification to address Ml stigma since then. ${ }^{17,18}$ The SDS is psychometrically sound and can be used to assess Ml stigma among all adults.

Measuring stigma objectively using a standardized tool is extremely meaningful. It provides valuable information about the scope and magnitude of the problem that can guide targeted programming efforts. Using the same scale over time is an effective and reliable way to measure progress and highlight shifts in patterns related to stigma along a continuum.

\section{Stigma reduction strategies}

Raising awareness through self-reflection is an important first step. Performing a quick self-check to expose unconscious prejudices that drive stigma-related behaviors can serve as a wake-up call and translate to public awareness. By recognizing personal bias, one will more likely be empowered to inform and educate others. ${ }^{19}$ Increasing public awareness about the staggering statistics related to self- and social stigma may be another plausible way to initiate the much needed change in attitudes and behaviors.

Education is an essential element to effectively reduce $\mathrm{Ml}$ stigma among providers and patients in the healthcare setting. Teaching healthcare personnel the skills needed to communicate and care for patients with $\mathrm{Ml}$ is integral. ${ }^{20}$ Providing training that includes first-hand testimonials from individuals with lived experiences has been found one of the most effective and transformational ways to increase knowledge, decrease stereotypes, and promote empathy. ${ }^{20-22}$ Direct social contact with those who have encountered stigma firsthand provides validation for patients with $\mathrm{Ml}$ and facilitates open dialogue between provider and patient. ${ }^{22-24}$

Stigma reduction strategies should also include myth debunking interventions that demonstrate how unconscious prejudices can negatively impact patient outcomes. ${ }^{20,25}$ Recovery should be emphasized, because this is the elusive yet attainable goal for all who suffer from MI. ${ }^{20}$ Although healthcare providers play a pivotal role in initiating steps to achieve this goal, research shows that many do not believe recovery is possible. ${ }^{14}$ Implementation of recovery-oriented practices, including goal setting, shared decision making, and establishment of a strong provider-patient partnership with mutual respect as the foundation, should be employed. ${ }^{26-29}$

Assigning equal value to mental and physical well-being is crucial. Consistent incorporation of a mental health check into the review of systems during routine encounters will convey a positive attitude and may help break the cycle of stigma. Opening the lines of communication in a nonjudgmental, caring manner can create a safe place for candid conversations regarding symptoms or concerns about MI. It is also important to recognize that everyone is susceptible to the effects of day-to-day pressures on mental health. Add on stress related to chronic illness, fearsome world events, or life circumstances beyond our control, and it is easy to recognize that no one is immune to this vulnerability. By cultivating and maintaining trusted patient-provider relationships, free of bias and prejudice, nurse practitioners (NPs) are well positioned to address MI before its deleterious effects ensue. Brief counseling or a referral to a mental health professional may be all that is needed to halt the development of MI or to expedite recovery.

\section{Conclusion}

Mental illness is highly prevalent in the United States and worldwide. Providers in all healthcare arenas and specialties are sure to encounter affected patients routinely in practice. In fact, the Centers for Disease Control and Prevention reports $45 \%$ of mental health-related office visits are conducted in primary or non-mental health specialty practices. ${ }^{30}$ Despite high prevalence, most women with Ml are unlikely to seek care from mental health professionals due to stigma and other social influences, but they may be inclined to discuss concerns in more familiar settings. ${ }^{1}$ Non-mental health providers are often the first providers to whom patients experiencing new or ongoing symptoms of Ml will turn. As frontline healthcare providers, NPs play a key role in bridging the gap to appropriate care. Women's health NPs are ideally positioned to decrease MI stigma and facilitate necessary mental health services as providers of women's healthcare across the lifespan. Personal and public awareness of potential barriers, namely stigma, is an important component in providing efficient care that integrates physical and mental health. Self-reflection, stigma assessment, and engagement in strategies to increase knowledge will enable NPs to partner with patients who suffer from Ml, empower them, and facilitate high-quality care that can lead to recovery.

Teresa M. Conklin is a family nurse practitioner and clinical instructor at Seton Hall University, South Orange, New Jersey. The author has no actual or potential conflicts of interest in relation to the contents of this article.

\section{References}

1. World Health Organization. Mental disorders affect one in four 
people. October 4, 2019. https:// www.who.int/whr/2001/media_ centre/press_release/en/.

2. National Institute of Mental Health. Mental illness. February 2019. https://www.nimh.nih.gov/health/ statistics/mental-illness.shtml.

3. American Psychiatric Association. Mental health disparities: women's mental health. December 19, 2017. Washington DC: American Psychiatric Association; 2017.

4. National Institute of Mental Health Suicide. April 2019. https://www. nimh.nih.gov/health/statistics/suicide.shtml.

5. American Psychiatric Association. Depression screening rates among adults increased slightly in recent years, but remain low. July 9 , 2018. https://www.psychiatry.org/ newsroom/news-releases/depression-screening-rates-among-adultsincreased-slightly-in-recent-yearsbut-remain-low.

6. Lake J, Turner MS. Urgent need for improved mental health care and a more collaborative model of care. Perm J. 2017;21:17-24.

7. National Council for Behavioral Health, Cohen Veteran Network. America's mental health 2018. October 10, 2018. https://www. thenationalcouncil.org/policy-action/what-is-the-state-of-americasmental-health/.

8. American Psychological Association. Mental health is a human right. December 2018. https:// www.apa.org/international/ pi/2018/12/mental-health-rights.

9. Stigma: Definition by Oxford Dictionary. Lexico Dictionaries, English. https://www.lexico.com/en/ definition/stigma.

10. Haddad P, Haddad I. Mental health stigma. British Association for Psychopharmacology Articles. August 15, 2016. https://www. bap.org.uk/articles/mental-healthstigma/.

11. Thornicroft G, Rose D, Kassam A, Sartorius N. Stigma: ignorance, prejudice or discrimination? $\mathrm{Br} \mathrm{J}$ Psychiatry. 2007;190:192-193.

12. Maharjan S, Panthee B. Prevalence of self-stigma and its association with self-esteem among psychiatric patients in a Nepalese teaching hospital: a cross-sectional study. BMC Psychiatry. 2019;19(1):347.

13. Seeman N, Tang S, Brown AD, Ing A. World survey of mental illness stigma. J Affect Disord. 2016;190:115-121.

14. Knaak S, Mantler E, Szeto A. Mental illness-related stigma in healthcare: barriers to access and care and evidence-based solutions. Healthc Manage Forum. 2017;30(2):111-116.

15. Luty J, Fekadu D, Umoh O, Gallagher J. Validation of a short instrument to measure stigmatised attitudes towards mental illness. Psychiatric Bull. 2006;30(7):257260.

16. Stuart H, Miley R, Koller M. The inventory of stigmatizing experiences: its development and reliability. World Psychiatry. 2005;4(1);33-37.

17. Bogardus E. Measuring social distance. J Applied Sociology. 1925;9:299-308.

18. Link BG, Phelan JC, Bresnahan M, et al. Public conceptions of mental illness: labels, causes, dangerousness, and social distance. $A m J$ Pub Health. 1999;89(9):1328-1333.

19. Sussman D. Five steps to reduce stigma about mental illness. Psychol Today. March 13, 2019. https://www.psychologytoday. com/us/blog/the-recoverycoach/201903/5-steps-reduce-stigma-about-mental-illness.

20. Knaak S, Modgill G, Patten SB. Key ingredients of anti-stigma programs for health care providers: a data synthesis of evaluative studies. Can J Psychiatry. 2014;59(10 suppl 1):S19-S26.

21. Corrigan PW, Morris SB, Michaels PJ, et al. Challenging the public stigma of mental illness: a metaanalysis of outcome studies. Psychiatr Serv. 2012;63(10):963-973.

22. Maranzan KA. Interprofessional education in mental health: an opportunity to reduce mental illness stigma. J Interprof Care. 2016;30(3):370-377.

23. Pettigrew TF, Tropp LR. A me- ta-analytic test of intergroup contact theory. J Pers Soc Psychol. 2006;90(5):751-783.

24. Agrawal S, Capponi P, López J, et al. From surviving to advising: a novel course pairing mental health and addictions service users as advisors to senior psychiatry residents. Acad Psychiatry. 2016;40(3):475-480.

25. Sukhera J, Chahine S. Reducing mental illness stigma through unconscious bias-informed education. MedEdPublish. 2016;5.

26. Ungar T, Knaak S, Szeto ACH. Theoretical and practical considerations for combating mental illness stigma in health care. Commun Ment Health J. 2016;52(3):262-271.

27. Bracken P, Thomas P, Timimi S, et al. Psychiatry beyond the current paradigm. Br J Psychiatry. 2012;201(6):430-434.

28. Duncan E, Best C, Hagen S. Shared decision-making interventions for people with mental health conditions. Cochrane Database Syst Rev. 2010(1):CD007297.

29. Mental Health Commission of Canada. Recovery Guidelines, 2015. Ottawa, ON: Mental Health Commission of Canada; 2015.

30. Centers for Disease Control and Prevention. Mental health-related office visits by adults aged 18 and over: United States, 2012-2014. June 2018. https://www.cdc.gov/ nchs/products/databriefs/db311. htm. 